Canadian Oncology

Nursing Journal

Revue canadienne

de soins infirmiers

en oncologie

Volume 28, Issue 1 • Winter 2018

elSSN: 2368-8076 


\title{
Évaluation de l'application du modèle synergique en hématologie pour améliorer la prestation des soins et l'environnement de travail
}

\author{
par Georgia Georgiou, Yayra Amenudzie, Enoch Ho et Elizabeth O'Sullivan
}

\section{RÉSUMÉ}

Une unité d'hématologie a mis sur pied un projet pilote pour évaluer les effets du modèle synergique (Curley, 1998) sur la prestation des soins aux patients et la pratique professionnelle. Selon ce modèle, le jumelage des infirmières et des patients dépend de la compétence des premières et des caractéristiques des seconds; l'acuité des soins se voit aussi attribuer une note, ce qui permet d'ajuster les affectations. Cette façon de faire donne lieu à de "meilleures correspondances » : $87 \%$ des infirmières ont affirmé que leurs compétences convenaient bien à l'acuité des soins des patients dont elles avaient la charge, comparativement à $48 \%$ avant la mise en place du modèle. L'amélioration touche également la satisfaction par rapport au soutien des infirmières novices, la participation aux affections, la charge de travail et l'engagement. On a, en outre, observé une diminution des incidents liés à la sécurité et une réduction des heures supplémentaires. Le modèle synergique se révèle un cadre prometteur pour optimiser l'environnement de travail et parfaire la prestation des soins pour d'autres populations de patients aux caractéristiques analogues.

\section{CONTEXTE}

드 n 2012, le programme d'hématologie et de greffes de cellucancérologie Juravinski a vu une occasion unique d'améliorer les soins aux patients et l'environnement de travail en mettant à l'essai le modèle synergique (Curley, 2007). Ce cadre de pratique professionnel est fondé sur l'évaluation de l'acuité des soins des patients, qui permet d'établir la bonne « correspondance » entre les besoins des patients et les compétences du

\section{AUX SUJET DES AUTEURES \\ Georgia Georgiou, B.A., M.Ed., gestionnaire clinique du programme de greffe de cellules souches, Unité d'hématologie Ron et Nancy Clark, Centre hospitalier et de cancérologie Juravinski, Centre des sciences de la santé d'Hamilton, 699, rue Concession, Hamilton (Ontario) L8V 5C2. T: 905-521-2100, poste 43910. Courriel : georgg@hhsc.ca \\ Yayra Amenudzie, inf. aut., M.Sc.inf., infirmière en hématologie, Unité d'hématologie Ron et Nancy Clark, Centre hospitalier et de cancérologie Juravinski, Centre des sciences de la santé d'Hamilton, 699, rue Concession, Hamilton (Ontario) L8V 5C2. 905-521-2100, poste 43910. \\ Courriel : amenudzie@hhsc.ca}

Enoch Ho, M.Pht., pht., acup., Bureau de l'amélioration de la qualité et de la valeur. Tél. : 905-521-2100, poste 42097. Courriel : hoenoch@HHSC.CA

Elizabeth O'Sullivan, inf. aut., B.Sc.inf., M.Sc.inf., directrice retraitée

DOI: $10.5737 / 236880762811721$ personnel et de procéder aux affectations en conséquence. Le travail d'équipe, la communication et l'affection des infirmières et des aides-soignants étaient problématiques dans l'unité. La plupart du temps, les affectations quotidiennes étaient déterminées par habitude et de manière intuitive, en tenant compte des aspects géographiques et du temps requis pour accomplir les différentes interventions. Par conséquent, les pratiques variaient d'un quart de travail à l'autre et d'une infirmière-chef à l'autre, amenant des frictions dans l'équipe et des plaintes sur la charge de travail et des affectations dangereuses. Tout cela influençait négativement le travail d'équipe, le moral du personnel, la qualité des soins aux patients, ainsi que la satisfaction, le recrutement et la rétention du personnel.

Dans l'éventail des solutions possibles, le modèle synergique s'est imposé comme un cadre prometteur. Il est censé permettre d'organiser efficacement les soins aux patients de manière à optimiser leur état de santé et à créer un milieu de travail attirant qui incitera les infirmières à venir y travailler et à y demeurer (Kaplow et Reed, 2008). MacPhee, Wardrop, Campbell et Wejr (2011) ont conclu que le modèle « ...aidait à identifier les infirmières les plus aptes à s'occuper des patients présentant des besoins particuliers; la philosophie du modèle, axée sur les patients, a rétabli la collaboration et le travail d'équipe en donnant à tous un objectif unifié, celui d’offrir de meilleurs soins. De plus, le modèle et les outils ont enseigné au personnel un langage commun, une approche systématique de pondération des soins ainsi que des lignes directives objectives en matière de dotation » (p. 52). Le modèle, déjà mis à l'épreuve dans un hôpital pour enfants, avait montré son efficacité pour améliorer la prestation des soins et l'état des patients (Gralton, 2012); il avait également permis d'augmenter la satisfaction des patients et le rendement des infirmières dans des unités de soins intensif en cardiologie (Khalifehzadeh, 2012).

Le modèle synergique a été appliqué dans différents milieux de soins (de courte durée, en établissement et communautaires) et auprès de populations de patients variées. Le modèle est conçu pour aider les fournisseurs de soins de santé à classer les soins des patients par ordre de priorité, à jauger la charge de travail associée à chaque cas, à établir une approche systématique des réunions d'étude et des conférences de cas, à former et à orienter les nouveaux employés, à prendre des décisions concernant la planification des traitements, à offrir les soins et à procéder aux affectations sur le vif en fonction des notes synergiques (MacPhee, Wardrop, Campbell et Wejr, 2011; Kerfoot et al., 2006; Swickard et al., 2015; Carter et Burnette, 2011). Toutefois, il semble que le modèle n'ait jamais été appliqué dans une unité d'hospitalisation de courte durée en hématologie. 


\section{BUT DE L'ÉTUDE}

Le but du projet pilote était d'examiner l'adaptabilité, l'applicabilité et l'efficacité du modèle synergique, au Canada, dans une unité d'hospitalisation en hématologie et greffes de cellules souches hématopoïétiques. Le projet a été mené en deux étapes sur une période de 18 mois.

La première phase visait à répondre aux questions suivantes :

- Le modèle synergique peut-il être adapté aux patients en hématologie?

- Est-il possible de mettre en place le modèle dans un milieu d'hospitalisation de courte durée?

- Quels sont les facteurs de succès déterminants et les limites du modèle?

Les résultats de la première étape ont confirmé que le modèle pouvait être adapté et mis en place avec succès auprès de patients en hématologie. Le Guide provincial du projet sur la charge de travail des infirmières, publié par la British Columbia Nurses' Union (2010), a été utilisé pour créer un outil d'évaluation de l'acuité des soins prodigués aux patients de notre population en fonction de quatre caractéristiques : la complexité, la stabilité, la participation aux soins et la prévisibilité. En outre, nous avons pu constater qu'il était possible d'implanter, en contexte canadien et dans un milieu d'hospitalisation de courte durée, les procédures élaborées pour noter les patients et pour décider, à l'aide de ces notes, de l'affectation des infirmières et de l'ajustement de la dotation en personnel. Les conclusions de la première phase du projet, notamment les leçons à tirer des principaux facteurs habilitants et des limites du modèles, ont déjà fait l'objet d'un autre article (Amenudzie, Georgiou, Ho et O'Sullivan, 2017).

Le présent article traite plutôt de la seconde étape du projet pilote, dont le but était d'évaluer l'utilité et les répercussions $\mathrm{du}$ modèle synergique sur l'environnement de pratique professionnelle et la prestation des soins. Il aborde la méthodologie employée pour établir l'utilité du modèle, les résultats, les recommandations à l'intention des administrateurs, ainsi que les orientations futures.

\section{MÉTHODOLOGIE}

Pour évaluer les effets du modèle sur l'environnement de travail et les pratiques professionnelles, on a invité toutes les infirmières à remplir un sondage sur la synergie dans l'environnement de travail, avant et après la mise en place du projet pilote. Le sondage proposé dans le Guide provincial du projet sur la charge de travail des infirmières (BCNU, 2010) a été adapté et utilisé pour mesurer différents aspects de l'environnement de pratique, notamment :

a) le soutien offert aux infirmières novices;

b) la disponibilité des infirmières-chefs d'expérience;

c) la capacité de se concentrer sur les tâches infirmières;

d) la capacité de prodiguer des soins infirmiers fondés sur des données probantes;

e) la collaboration entre les infirmières et d'autres professionnels de la santé dans la prise de décision;

f) la capacité de planifier les réunions d'étude de cas et d'y prendre part; g) la participation à l'attribution des ressources et aux décisions concernant leur utilisation;

h) la participation à l'affectation du personnel;

i) le niveau de correspondance entre les affectations et les compétences des infirmières, permettant à ces dernières d'offrir des soins de qualité, sûrs et éthiques;

j) l'orientation des nouveaux employés;

k) les possibilités de formation continue, de formation en cours d'emploi, ainsi que de réunions entre collègues et de réflexion sur la pratique;

1) la mesure dans laquelle l'environnement de travail permet aux infirmières d'établir une relation thérapeutique satisfaisante avec les patients.

Les notes attribuées avant et après la mise en place du modèle ont été comparées à l'aide du test du khi carré pour en déterminer la signification statistique $(\mathrm{p}<.05)$.

Les effets du modèle sur la satisfaction des infirmières par rapport aux affectations et au degré de correspondance entre leurs compétences et les caractéristiques des patients ont été évalués grâce à un sondage, rempli quotidiennement par les infirmières pendant plus de 73 quarts de travail de jour, soit du 13 janvier 2014 au 31 mars 2014. À la fin de chaque quart de travail, les infirmières devaient préciser les éléments suivants :

- leur niveau de compétence (infirmière novice, débutante avancée, compétente, très compétente, experte);

- le rythme et la charge de travail (rythme de travail idéal permettant d'aller au-delà des soins de routine; rythme exigeant mais gérable; rythme exigeant et difficile à gérer; rythme impossible à gérer et dangereux);

- la correspondance entre les notes de synergie, les besoins des patients et l'acuité des soins requis;

- le degré de correspondance entre les compétences des infirmières et l'acuité des soins requis;

- tout autre commentaire sur des points en lien avec les affectations.

Pour déterminer les répercussions du modèle sur l'engagement du personnel, on a posé aux infirmières quatre questions tirées d'un sondage mené en 2011 dans tout l'hôpital et qui portait justement sur ce thème. Les questions mesuraient la perception du personnel par rapport aux éléments suivants : le sentiment d'engagement, la qualité des soins aux patients, l'équilibre entre les impératifs professionnels et personnels et le ratio de compensation stress/satisfaction (Stress Satisfaction Offset Score). Ces questions ont été incluses au sondage d'évaluation réalisé après l'application du modèle synergique, à la fin du projet pilote. Les réponses du sondage original pan-hospitalier ont été filtrées pour conserver uniquement les notes attribuées à l'engagement par les infirmières avant et après la mise en place du modèle; ces notes ont ensuite été comparées à l'aide du test du khi carré.

Pour explorer les répercussions du modèle sur les indicateurs relatifs au travail infirmier tels que les chutes des patients, les erreurs de médication et autres incidents liés à la sécurité, des données ont été extraites de la base de données des incidents liés à la sécurité. Le nombre de cas survenus avant le 
projet pilote (entre novembre 2012 et mars 2013) et après (de novembre 2013 à mars 2014) ont par la suite été comparés.

Enfin, pour évaluer les effets du modèle sur l'utilisation des ressources, on a observé le nombre de quarts de travail où des infirmières ou autres fournisseurs de soins supplémentaires ont dû venir prêter main-forte. Le nombre d'heures supplémentaires mensuelles pendant la période d'avril 2012 à mars 2013 est extrait de la base de données de l'hôpital.

\begin{tabular}{|l|l|l|l|}
\hline \multicolumn{4}{|c|}{ Tableau 1: Perception des infirmières par rapport aux affectations } \\
\hline $\begin{array}{l}\text { Note accordée par } \\
\text { les infirmières aux } \\
\text { affectations }\end{array}$ & $\begin{array}{l}\text { AVANT LA } \\
\text { SYNERGIE } \\
\text { N = } 127\end{array}$ & $\begin{array}{l}\text { APRÈS LA } \\
\text { SYNERGIE } \\
\text { N=568 }\end{array}$ & $\begin{array}{l}\text { Test du } \\
\text { khi carré }\end{array}$ \\
\hline $\begin{array}{l}\text { La majorité (80 \%) des } \\
\text { infirmières d'un même } \\
\text { quart ont qualifié leur } \\
\text { affectation "d'idéale " } \\
\text { ou " d'exigeante mais } \\
\text { gérable " }\end{array}$ & $53 \%$ & $70 \%$ & $p<.05$ \\
\hline
\end{tabular}

\section{RÉSULTATS}

Le projet pilote a eu lieu d'octobre 2013 à mars 2014. En comparant les mesures réalisées avant et après la mise à l'essai du modèle, on a constaté des améliorations statistiquement significatives dans différents domaines.

D'abord, pour ce qui est de l'utilité de l'outil de synergie (voir les tableaux 1 et 2 ), les résultats indiquent une meilleure « correspondance » entre les besoins des patients et les compétences des infirmières. Les infirmières novices se sentaient mieux appuyées dans leur pratique et, de l'avis des infirmières, l'affectation des patients et la charge de travail associée était plus facilement gérable. Les infirmières utilisaient avec diligence l'outil de pondération de l'acuité des soins, de même que l'outil d'organisation des affectations quotidiennes et les lignes directrices en matière de dotation. Essentiellement, les notes de synergie rendaient compte avec exactitude de l'état de santé des patients.

Côté engagement (voir le tableau 3), on a observé un engagement plus actif de la part des infirmières, une plus grande participation aux décisions, ainsi qu'une perception plus

Tableau 2 : Perception des infirmières par rapport au milieu de pratique et à l'utilité de l'outil

\section{SONDAGE AUPRÈS DES INFRIMIÈRES - \% de notes positives}

\begin{tabular}{|l|l|l|}
\hline $\begin{array}{l}\text { AVANT LA SYNERGIE } \\
\text { N=29 Taux de réponse } \\
\text { de } 53 \%\end{array}$ & $\begin{array}{l}\text { APRĖS LA SYNERGIE } \\
\text { Ne32 Taux de réponse } \\
\text { de } 60 \%\end{array}$ & $\begin{array}{l}\text { Test du } \\
\text { khi carré }\end{array}$ \\
\hline $17 \%$ & $65 \%$ & $p<.05$ \\
\hline $48 \%$ & $87 \%$ & $p<.05$ \\
\hline $55 \%$ & $87 \%$ & $p<.05$ \\
\hline $100 \%$ & $65 \%$ & $p<.05$ \\
\hline $100 \%$ & & \\
\hline $76 \%$ & & \\
\hline $94 \%$ & & \\
\hline
\end{tabular}

Tableau 3 : Perceptions des infirmières par rapport à l'engagement

\begin{tabular}{|l|l|l|l|}
\hline $\begin{array}{l}\text { Résultats du sondage sur l'engagement du personnel (pourcentage de } \\
\text { notes positives) }\end{array}$ & $\begin{array}{l}\text { AVANT LA SYNERGIE } \\
\text { Taux de réponse de 46\% }\end{array}$ & $\begin{array}{l}\text { APRÈS LA SYNERGIE } \\
\text { Taux de réponse de } \\
60 \%\end{array}$ & $\begin{array}{l}\text { Test du } \\
\text { khi carré }\end{array}$ \\
\hline Perception du sentiment d'engagement & $43 \%$ & $62 \%$ & $p<.05$ \\
\hline Perception de la qualité des soins aux patients & $62 \%$ & $76 \%$ & $p<.05$ \\
\hline $\begin{array}{l}\text { Perception de la charge de travail et de l'équilibre entre vie } \\
\text { professionnelle et personnelle }\end{array}$ & $24 \%$ & $50 \% .05$ & $56 \%$ \\
\hline Ratio de compensation stress/satisfaction (Stress Satisfaction Offset Scores) & $26 \%$ & $p<.05$ \\
\hline
\end{tabular}


positive de la qualité des soins. Les infirmières disaient bénéficier d'un meilleur équilibre entre leur vie personnelle et professionnelle.

Ensuite, le modèle a permis de réduire le nombre d'incidents liés à la sécurité ou aux erreurs (voir le tableau 4), principalement des chutes des patients et des erreurs de laboratoire.

Enfin, en ce qui concerne l'effet sur l'utilisation des ressources (voir les tableaux 5 et 6 ), on a dû faire appel à des aides-soignants ou à des infirmières surnuméraires pour 27 et 23 quarts de travail respectivement. Les heures supplémentaires ont diminué de $40 \%$ et les variations mensuelles étaient moindres.

\section{DISCUSSION}

L'expérience réalisée par notre équipe avec le modèle synergique et les résultats du projet pilote permettent de confirmer les promesses du modèle pour l'amélioration de la prestation des soins et de l'environnement de travail. Grâce au modèle, l'équipe pouvait assigner l'infirmière la plus apte à dispenser les soins aux patients. La collaboration et le travail d'équipe ont progressé, notre équipe a acquis un langage commun pour discuter de l'acuité des soins et des besoins des patients, et nous avons adopté une approche systématique des affectations et des décisions en matière de dotation. Notre expérience avec le modèle synergique a non seulement forgé notre manière de prodiguer les soins, d'utiliser les ressources et d'organiser la dotation en personnel, mais elle a aussi transformé nos modes d'évaluation des besoins en perfectionnement des infirmières, ainsi que nos méthodes d'orientation et de formation des nouveaux employés. L'évaluation des compétences infirmières fait partie intégrante de l'évaluation du rendement et du perfectionnement professionnel; le profil des compétences permet de répartir les nouvelles infirmières dans les différentes rotations afin d'optimiser la représentation des compétences pour chaque quart de travail.

\section{Implications pour les administrateurs}

Le Rapport sur la santé dans le monde de 2006, ainsi que les recherches portant sur les hôpitaux-aimants soulignent l'importance de créer un environnement de travail sain (Havens et Aiken, 1999; Shamian et El-Jardali, 2007). Il existe un lien

Tableau 4 : Répercussions sur les incidents liés à la sécurité et les erreurs

\begin{tabular}{|l|l|}
\hline $\begin{array}{l}\text { Avant la synergie De } \\
\text { novembre } 2012 \text { à mars } 2013\end{array}$ & $\begin{array}{l}\text { Après la synergie De } \\
\text { novembre } 2013 \text { à mars } 2014\end{array}$ \\
\hline 144 incidents liés à la sécurité & 107 incidents liés à la sécurité \\
\hline *Réduction du nombre de chutes et d'erreurs de laboratoire \\
\hline
\end{tabular}

\section{Tableau 5 : Utilisation des ressources}

\begin{tabular}{|l|l|}
\hline Type de ressources & $\begin{array}{l}\text { Nombre de quarts de travail et d'heures } \\
\text { (de janvier 2014 à mars 2014) }\end{array}$ \\
\hline Aides-soignants & 27 quarts de travail, 303,75 heures \\
\hline Infirmières & 23 quarts de travail, 258,75 heures \\
\hline
\end{tabular}

étroit entre un milieu de travail agréable, la satisfaction des infirmières et la qualité des soins (Armstrong et Laschinger, 2006; Laschinger et al., 2003). L'une des composantes importante d'un milieu de travail sain est la participation des infirmières de première ligne à la prise de décisions concernant le modèle de prestation des soins. L'application du modèle synergique dans notre unité a accru l'autonomie clinique et l'engagement des infirmières en les faisant participer aux décisions influençant le soin des patients, la qualité du milieu de travail et l'utilisation des ressources. Nos résultats montrent que les leaders peuvent avoir une influence positive sur l'environnement de pratique en adoptant un cadre philosophique, structurel et procédural qui encourage les infirmières à agir sur leur milieu de pratique et qui les incite à exercer leur jugement professionnel pour prendre des décisions concernant les patients ou l'unité.

De plus, le modèle synergique s'harmonise bien avec plusieurs normes des services de traitement du cancer et d'oncologie d'Agrément Canada. En l'adoptant, l'organisation peut faire la preuve qu'elle a) offre du soutien à l'équipe dans sa prestation de soins de qualité aux clients; b) s'est dotée de critères pour assurer l'affectation juste et équitable des membres de l'équipe aux patients; c) donne aux membres de l'équipe la possibilité d'exprimer leur avis sur le travail et la conception des tâches.

Enfin, le modèle synergique peut aisément servir à évaluer la compétence des infirmières et à soutenir le perfectionnement professionnel, contribuant ainsi à créer un environnement de travail propice à la rétention des effectifs.

\section{Orientations futures pour la recherche}

Bien que le projet pilote ait permis une réduction des heures supplémentaires et des incidents liés à la sécurité, ainsi qu'une amélioration de l'engagement et de la satisfaction des infirmières, il est impossible, vu l'interdépendance et la complexité des activités cliniques, d'attribuer les changements positifs au seul modèle synergique, d'autant plus que sa mise en œuvre s'est faite en parallèle avec

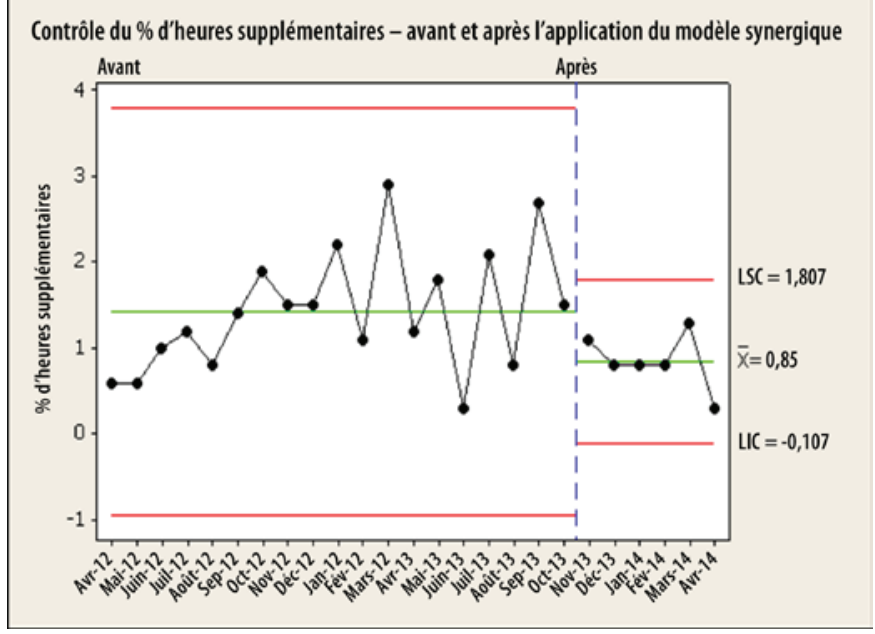

Tableau 6 : Pourcentage de temps supplémentaire 
d'autres interventions visant à enrichir l'environnement de travail. D'autres recherches seront nécessaires pour examiner les répercussions et le rapport coût-efficacité du modèle à la lumière des résultats propres au personnel, aux patients et à l'organisation, par exemple l'absentéisme, le roulement de personnel, les incidents liés à la sécurité, la satisfaction des patients et les indicateurs cliniques relatifs au travail infirmier. Enfin, il pourrait être intéressant de mettre au point une note globale qui rendrait compte du niveau général d'acuité des soins dans l'unité. Cela permettrait d'accorder les différents domaines cliniques aux tendances observées et peut-être de simplifier les processus permettant de décider s'il convient ou non d'ajuster la dotation en personnel.

\section{CONCLUSION}

Dans notre unité, le modèle synergique est devenu le système opérationnel d'organisation du travail des infirmières et des aides-soignants. Il propose un cadre efficace pour améliorer la pratique professionnelle et promouvoir un

\section{RÉFÉRENCES}

Amenudzie, Y., Georgiou, G., Ho, E., \& O’Sullivan, E. (2017). Adapting and applying the Synergy Model on an inpatient heamatology unit. Canadian Oncology Nursing Journal, 27(4), 338-342.

Armstrong, K., \& Laschinger (2006). Structural empowerment, magnet hospital characteristics, and patient safety culture: Making the link. Journal of Nursing Care Quality, 21(2), 124-132.

British Columbia Nurses' Union. (2010). Provincial nursing workload project resource toolkit for teams: Nursing workload and staffing plan processes. https://www.bcnu.org/Safeworkplace/ DefendProfessionalPractice/Documents/pnwp_resource_toolkit.pdf

Carter, K.F., \& Burnette, H.D. (2011). Creating patient-nurse synergy on a medical-surgical unit. Medsurg Nursing, 20(5), 249-254.

Curley, M.A. (Ed.). (2007). Synergy: The unique relationship between nurses and patients. Indianapolis: Sigma Theta Tau International.

Curley, M.A. (1998). Patient-nurse synergy: Optimizing patient's outcomes. American Journal of Critical Care, 7(1), 64-72.

Gralton, K.S., \& Brett, S.A. (2012). Integrating the synergy model for patient care at Children's Hospital in Wisconsin. Journal of Pediatric Nursing, 27, 74-81.

Havens, D., \& Aiken, L. (1999). Shaping systems to promote outcomes: The magnet hospital model. Journal of Nursing Administration, 29, 14-20.

Kaplow, R., \& Reed, K. (2008). The AACN synergy model for patient care: A nursing model as a force of magnetism. Nursing Economics, 26(1), 17-25. environnement de travail sain. Le modèle a optimisé la pratique infirmière en établissant des balises claires pour le perfectionnement professionnel et le jumelage des compétences infirmières et des besoins uniques des patients. La nature souple du modèle convient bien à l'acuité variable des soins à prodiguer aux patients en hématologie. Cette souplesse permet à l'équipe de soins et aux administrateurs de prendre, sur le vif, des décisions mieux éclairées concernant l'utilisation et l'attribution des ressources afin de s'adapter aux fluctuations de l'acuité des soins.

\section{REMERCIEMENTS}

Nous désirons remercier l'équipe de projet, le personnel et les médecins de l'unité d'hématologie et de greffes de cellules souches hématopoiétiques, ainsi que la Fondation du Centre de cancérologie Juravinski et F. Hoffmann-La Roche Ltd., qui ont apporté le financement et le soutien nécessaire à la réalisation du projet pilote.

Kerfoot, K.M., Lavandero, R., Cox, M., Triola, N., Pacinin, C. \& Hanson, D.M. (2006). Conceptual models and the nursing organization. Implementing the AACN synergy model for patient care. Nurse Leader, 20-26.

Khalifehzadeh, A., Jahromi, M.K., \& Yazdannik, A. (2012). The impact of the Synergy Model on nurses' performance and the satisfaction of patients with acute coronary syndrome. Iranian Journal Nursing and Midwifery Research, 17(1), 16-20.

Laschinger, H., Almost, J., \& Tuer-Hodes. (2003). Workplace empowerment and magnet hospital characteristics. Journal of Nursing Administration, 33(7/8), 410-422.

MacPhee, M.K., Jewell, K., Wardrop, A., Ahmed, A., \& Mildon, B. (2010). British Columbia's provincial nursing workload project: Evidence to empowerment. Canadian Journal of Nursing Leadership, 23(1), 54-63.

MacPhee, M., Wardrop, A., Campbell, C., \& Wejr, P. (2011). The synergy professional practice model and its patient characteristics tool: A staff empowerment strategy. Nursing Leadership, 24(3), $42-55$.

Shamian, J., \& El-Jardali, F. (2007). Healthy workplaces for health workers in Canada: Knowledge transfer and uptake in policy and practice. Healthcare Papers, 7(Special Issue), 6-25.

Swickard, S., Swickard, W., Reimer, A., Lindell, D., \& Winkelman, C. (2015). Adaptation of the AACN synergy model for patient care to critical care transport. Critical Care Nursing, 34(1), 16-28. 